\title{
OPEN A pre-formulation study of tetracaine loaded in optimized nanostructured lipid carriers
}

\author{
Simone R. Castro ${ }^{1}$, Lígia N. M. Ribeiro ${ }^{1,2}{ }^{2}$ Márcia C. Breitkreitz ${ }^{3}$, Viviane A. Guilherme ${ }^{1}$, \\ Gustavo H. Rodrigues da Silva ${ }^{1}$, Hery Mitsutake ${ }^{3}$, Ana C. S. Alcântara ${ }^{4}$, Fabiano Yokaichiya ${ }^{5}$, \\ Margareth K. K. D. Franco ${ }^{6}$, Daniel Clemens ${ }^{5}$, Ben Kent ${ }^{5}$, Marcelo Lancellotti ${ }^{7}$, \\ Daniele R. de Araújo ${ }^{8}$ \& Eneida de Paula ${ }^{1 \bowtie}$
}

Tetracaine (TTC) is a local anesthetic broadly used for topical and spinal blockade, despite its systemic toxicity. Encapsulation in nanostructured lipid carriers (NLC) may prolong TTC delivery at the site of injection, reducing such toxicity. This work reports the development of NLC loading $4 \%$ TTC. Structural properties and encapsulation efficiency (\%EE $>63 \%)$ guided the selection of three pre-formulations of different lipid composition, through a $2^{3}$ factorial design of experiments (DOE). DLS and TEM analyses revealed average sizes $(193-220 \mathrm{~nm})$, polydispersity $(<0.2)$, zeta potential $\mid-21.8$ to $-30.1 \mathrm{mV} \mid$ and spherical shape of the nanoparticles, while FTIR-ATR, NTA, DSC, XRD and SANS provided details on their structure and physicochemical stability over time. Interestingly, one optimized pre-formulation (CP-TRANS/TTC) showed phase-separation after 4 months, as predicted by Raman imaging that detected lack of miscibility between its solid (cetyl palmitate) and liquid (Transcutol) lipids. SANS analyses identified lamellar arrangements inside such nanoparticles, the thickness of the lamellae been decreased by TTC. As a result of this combined approach (DOE and biophysical techniques) two optimized pre-formulations were rationally selected, both with great potential as drug delivery systems, extending the release of the anesthetic ( $>48 \mathrm{~h}$ ) and reducing TTC cytotoxicity against Balb/c $3 T 3$ cells.

Local anesthetics prevent pain by interrupting nerve excitation and conduction. They interact with voltage-gated sodium channels, blocking the propagation of nerve impulses ${ }^{1,2}$. Tetracaine (TTC) is a potent anesthetic of the amino ester class, which fast metabolism by plasma esterases and high systemic toxicity ${ }^{3}$ have restricted its use to topical anesthesia in ophthalmic procedures, oropharyngeal pain, skin anesthesia and spinal block ${ }^{2,4,5}$.

In order to enhance the therapeutic effect and reduce the toxicity of local anesthetics, drug delivery systems (DDS) have shown promising results, in nanocarriers such as liposomes, cyclodextrins, polymeric particles ${ }^{6}$, solid lipid nanoparticles ${ }^{7,8}$, and nanostructured lipid carriers (NLC) ${ }^{9,10}$. NLC are colloidal systems, which consist of a lipid matrix composed of a mixture of solid and liquid (oils) lipids, plus a surfactant. The lipid matrix is a pseudo-crystalline structure with many imperfections ${ }^{11}$ that confer superior physicochemical stability to the nanoparticle and allow drug insertion, increasing its solubility, permeability, bioavailability and reducing adverse effects $^{12}$. In comparison to other lipid-based carriers (e.g. liposomes, solid lipid nanoparticles), NLC have longer shelf stability and higher upload capacity for lipophilic drugs ${ }^{10}$.

Here we report the development, among different lipid matrices, of novel DDS for tetracaine. Factorial design was used as a chemometric tool to elucidate the effect of excipients in the NLC supramolecular organization, providing robust systems with optimized structural properties for subsequent analyses ${ }^{13}$. Among other biophysical techniques, Raman imaging and SANS analyses disclosed details on the lipid matrix organization, that reduced the shelf stability of one optimized formulation. Two pre-formulations were then selected, aiming at improving

\footnotetext{
${ }^{1}$ Department of Biochemistry and Tissue Biology, Institute of Biology, University of Campinas (UNICAMP), Rua Monteiro Lobato 255, Campinas, SP 13083862, Brazil. 'Institute of Biotechnology, Federal University of Uberlandia, Uberlandia, MG, Brazil. ${ }^{3}$ Department of Analytical Chemistry, Institute of Chemistry, UNICAMP, Campinas, SP, Brazil. "Department of Chemistry, Federal University of Maranhão, São Luís, MA, Brazil. ${ }^{5}$ Department of Quantum Phenomena in Novel Materials, Helmholtz-Zentrum, Berlin, Germany. ${ }^{6}$ Nuclear and Energy Research Institute, IPEN-CNEN/SP, São Paulo, SP, Brazil. ${ }^{7}$ Faculty of Pharmaceutical Sciences, UNICAMP, Campinas, SP, Brazil. ${ }^{8}$ Human and Natural Science Center, Federal University of ABC (UFABC), Santo André, SP, Brazil. ${ }^{\circledR}$ email: depaula@unicamp.br
} 


\begin{tabular}{|l|l|l|l|}
\hline \multirow{2}{*}{ Independent variables } & \multicolumn{3}{l|}{ Levels } \\
\cline { 2 - 5 } & $\mathbf{- 1}$ & $\mathbf{0}$ & $\mathbf{+ 1}$ \\
\hline Surfactant concentration (\% P68 w/v) & 2 & 3.5 & 5 \\
\hline Lipid phase concentration (\% TL w/v) & 16 & 18 & 20 \\
\hline Solid:liquid lipid ratio (\% SL:LL w/w) & $70: 30$ & $80: 20$ & $90: 10$ \\
\hline Dependent variables & & Goal & \\
\hline Particle size (nm) & & $\begin{array}{l}\text { Mini- } \\
\text { mum }\end{array}$ & \\
\hline Polydispersity index (PDI) & & $\begin{array}{l}\text { Mini- } \\
\text { mum }\end{array}$ \\
\hline Zeta potential $(|\mathrm{mV}|$ ) & & $\begin{array}{l}\text { Maxi- } \\
\text { mum }\end{array}$ & \\
\hline
\end{tabular}

Table 1. Experimental variables and their levels, responses and optimization goals for the $2^{3}$ experimental designs of CP-TRANS, CP-DK and MM-DK nanostructured lipid carriers.

TTC local anesthetic action (CP-DK/TTC and MM-CK/TTC) for further application in clinics. They showed excellent shelf-stability ( $>1$ year), high upload capacity $(>60 \%)$, prolonged drug release $(>48 \mathrm{~h})$ and reduced cytotoxicity against Balb/c 3T3 fibroblasts.

\section{Materials and methods}

Tetracaine base (TTC) was purchased from AK Scientific, Inc. (Union City, USA). The surfactants Pluronic F68 (P68) and Tween 80 were supplied by Sigma (St. Louis, USA); cetyl palmitate (CP) and Dhaykol 6040 (DK) - or capric/caprylic triglycerides-by Dhaymers Quím. Fina (Taboão da Serra, Brazil); myristyl myristate (MM) by Croda (Campinas, Brazil) and diethylene glycol monoethyl ether or Transcutol (TRANS) by Gattefossé (Lyon, France). HPLC-grade acetonitrile and methanol were purchased from J.T. Baker (Allentown, USA). 3T3 cells were from American type culture collection (ATCC, Manassas, VA, USA). 3-(4,5-dimethylthiazol-2-yl)-2,5-diphenyltetrazolium bromide (MTT), RPMI medium, fetal bovine serum, penicillin, streptomycin and uranyl acetate were purchased from Sigma-Aldrich (Missouri, USA). All other reagents were of analytical grade. Deionized water $(18 \mathrm{M} \Omega$ ) was obtained from an Elga USF Maxima ultra-pure Water purifier.

NLC preparation. NLC of different matrices (CP-TRANS, CP-DK and MM-DK) were obtained by emulsification-ultrasonication ${ }^{14}$. The oily phase was prepared by melting solid and liquid lipids in a water bath, $10{ }^{\circ} \mathrm{C}$ above the melting temperatures of the solid lipid (42.6 and $55.0^{\circ} \mathrm{C}$ for MM and CP, respectively $)^{8,15}$. TTC was added to the mixture of melted lipids and stirred until complete solubilization. Simultaneously, an aqueous phase was prepared by dissolving the surfactant (P68) in heated deionized water. The aqueous phase was then poured into the lipid phase under stirring at 10,000 rpm (Ultra-Turrax T18, IKA T18 basic) for $3 \mathrm{~min}$. The obtained microemulsion was sonicated in a tip sonicator (Vibracell, Sonics \& Materials Inc.) at $500 \mathrm{~W}$ and $20 \mathrm{kHz}$ for $30 \mathrm{~min}$, in alternating on/off $30 \mathrm{~s}$ cycles. The resulting nanoemulsion was immediately cooled to room temperature in an ice bath to produce the NLC.

Experimental design. Three $2^{3}$ factorial designs were carried out, with triplicates at the central point, for each of the three lipid combinations: CP-TRANS, CP-DK and MM-DK. Experimental data were processed using Design-Expert software (version 9.0, State-Ease Inc., Minneapolis, USA). Analysis of variance (ANOVA) was applied to evaluate the significance of the effects, their interactions, and the lack of adjustment of the regression model, considering the confidence level of $95 \%(\alpha=0.05)$. Three independent variables were used: (a) surfactant concentration (\% P68), (b) total lipid concentration (\% TL), and (c) solid:liquid lipid ratio (expressed as \% SL), eleven NLC formulations (of nine different compositions) containing 4\% TTC were prepared in each of the $2^{3}$ factorial design. Table 1 shows the variables and levels used in this study, for each lipid combination.

The composition of the eleven formulations prepared according to the factorial design are shown in Table S1. They were all prepared in a random order and the central point triplicates encompassed all the sample preparation steps (authentic replicates).

Determination of particle size, polydispersity, zeta potential, concentration and $\mathrm{pH}$. The particle size (hydrodynamic diameter) and polydispersity index (PDI) were determined by dynamic light scattering (DLS) and zeta potential (ZP) by electrophoretic mobility, in a Nano ZS90 analyzer (Malvern Instruments, UK), at $25^{\circ} \mathrm{C}$. The samples were diluted $(1000 \times)$ in deionized water. The $\mathrm{pH}$ of NLC formulations were measured with a R-TEC7 $\mathrm{pH}$ meter (Tecnal Equip. Cient., Piracicaba, Brazil), at $25^{\circ} \mathrm{C}(\mathrm{n}=3)$.

The concentration of nanoparticles in the formulations was determined by Nanotracking analysis (NTA) in a NS300 (NanoSight, Amesbury, UK) equipment. The samples were diluted in deionized water $(5000 \times)$ and injected into the sample chamber with syringes. All measurements were performed at $25^{\circ} \mathrm{C},(\mathrm{n}=3)$.

Quantification of tetracaine by HPLC. TTC quantification, in triplicate, was performed in a Varian ProStar high-performance liquid chromatograph (HPLC) equipped with a PS 325 UV-Vis detector, PS 210 sol- 
vent delivery module and Galaxy Workstation software for data collection. A Luna $(5 \mu, 250 \times 4.6 \mathrm{~mm})$ reversephase C18 column (Phenomenex, Torrance, US) was used. Isocratic elution was run with a mobile phase of ammonium phosphate $(10 \mathrm{mM}, \mathrm{pH} 3.0)$ : acetonitrile, 70:30 v/v. The flow rate, injection volume and wavelength detection were set at $1.5 \mathrm{~mL} / \mathrm{min}, 30 \mu \mathrm{L}$ and $280 \mathrm{~nm}$, respectively. TTC retention time was $=5.3 \pm 0.1 \mathrm{~min}$. The assay method was validated, and the calibration curve was linear $\left(\mathrm{r}^{2}=0.9998\right)$ within the concentration range of 0.03-1.2 mM. The limits of detection and quantification of TTC were $4.47 \times 10^{-5} \mathrm{mM}$ and $1.49 \times 10^{-4} \mathrm{mM}$, respectively.

Encapsulation efficiency and drug loading determination. TTC encapsulation by the three NLC formulations (CP-TRANS/TTC, CP-DK/TTC and MM-DK/TTC) was determined in triplicate, by centrifugation-ultrafiltration, using $10 \mathrm{kDa}$ pore size Amicon regenerated cellulose filters (Millipore Corp., Bedford, USA). The samples were diluted in deionized water and centrifuged at $6000 \times g$ for $20 \mathrm{~min}$. The filtered aqueous solution was collected and free tetracaine was quantified at $280 \mathrm{~nm}$, by HPLC. The amount of TTC loaded in the NLC was expressed in terms of encapsulation efficiency (\%EE), calculated according to Eq. (1), or drug loading capacity (\%DL), accordingly to Eq. $(2)^{16}$ :

$$
\begin{gathered}
\% \mathrm{EE}=\frac{T T C_{\text {total }}-T T C_{\text {free }}}{T T C_{\text {total }}} \times 100 \\
\% \text { Drug loading }=\frac{\text { weight of encapsulated TTC }}{\text { weight of nanoparticles }} \times 100
\end{gathered}
$$

Transmission electron microscopy (TEM) images. The morphological analysis of the nanoparticles in all the three systems and their respective controls (prepared without TTC) was performed by transmission electron microscopy. 2\% Uranyl acetate was added to the diluted samples, for contrast. Aliquots of the samples were then deposited on copper grids coated with carbon film and dried at room temperature. After drying, micrographs of the samples were obtained in a JEOL 1200 EXII microscope, at $60 \mathrm{kV}$ and the images were edited with ImageJ software v.1.52a (https://imagej.nih.gov/ij/).

Infrared spectroscopy measurements (ATR-FTIR). ATR-FTIR analyses were performed for excipients, lyophilized formulations containing 4\% TTC (CP-TRANS/TTC, CP-DK/TTC and MM-DK/TTC) and their respective controls (CP-TRANS, CP-DK, MM-DK) without the anesthetic. Spectra were recorded in FTIR spectrophotometers (Bruker IFS $66 \mathrm{v} / \mathrm{S}$ or Perkin Elmer Spectrum 65) equipped with ATR cells, in the reflection mode, between 4500 and $500 \mathrm{~cm}^{-1}$.

Differential scanning calorimetry (DSC) analysis. DSC measurements of TTC, lyophilized NLC formulations (CP-TRANS/TTC, CP-DK/TTC, MM-DK/TTC), their controls (CP-TRANS, CP-DK, MM-DK), and solid lipid excipients (CP, MM) were taken in a TA Q20 calorimeter (Thermal Analysis Instruments, New Castle, USA) equipped with a cooling system. The samples $(5 \mathrm{mg})$ were placed in aluminum pans and the thermal profiles were obtained in the range of $0-250^{\circ} \mathrm{C}$, at a heating rate of $10^{\circ} \mathrm{C} / \mathrm{min}$, under nitrogen flow.

X-ray diffraction (XRD) analysis. X-ray powder diffraction (XRD) data were obtained in a Shimadzu XRD7000 diffractometer (Tokyo, Japan), using a Cu-Ka source at a scanning step of $2^{\circ} \mathrm{min}^{-1}$, between values of $2 \theta\left(5^{\circ}-50^{\circ}\right)$. Samples of lyophilized NLC containing 4\% TTC (CP-TRANS/TTC, CP-DK/TTC, MM-DK/TTC), their controls (CP-TRANS, CP-DK, MM-DK), and solid lipid excipients (CP, MM) were analyzed.

Small Angle Neutron Scattering (SANS) analysis. SANS measurements were performed at the VSANS-V16 (time of flight/very small angle scattering) instrument at Helmholtz-Zentrum Berlin (Germany). The scattering data was gathered at two sample detector distances: $1.7 \mathrm{~m}$ with neutron wavelengths of 1.8-3.8 $\AA$, and $11 \mathrm{~m}$ with 1.6-9.2 $\AA$ neutron wavelengths. The samples were placed in cuvettes (Hellma 110 QS) of $1 \mathrm{~mm}$ light path arranged in a sample holder that provides twenty positions per measurement. A waiting time of $30 \mathrm{~min}$ between measurements ensured the stabilization of the sample's temperature. Samples of NLC formulations containing 4\% tetracaine (CP-TRANS/TTC, CP-DK/TTC, MM-DK/TTC) and their controls (CP-TRANS, CP-DK, MM-DK), were prepared in $\mathrm{D}_{2} \mathrm{O}$, and measured at 25 and $37^{\circ} \mathrm{C}$. The final data reduction included corrections for sample transmission, background detector counts, empty cell scattering and detector efficiency, and they were scaled to absolute intensity using a $1 \mathrm{~mm} \mathrm{H}_{2} \mathrm{O}$ standard measurement. The SANS data were radially averaged and combined to give a total $q$ range of $0.005-0.5 \AA^{-1}{ }^{17}$. To gain a further insight about the nanoparticles containing DK (for which hydrophobic clusters between SL-LL were observed), their SANS data were modelled using the empirical function shown in Eq. (3):

$$
I(q)=\frac{A}{q^{n}}+\frac{B}{1+(q \xi)^{m}}+\text { back }
$$

where $\mathrm{A}$ and $\mathrm{B}$ are constants, $\mathrm{n}$ and $\mathrm{m}$ are power-law indices, back refers to the incoherent background and $\xi$ is the correlation length ${ }^{17-24}$. The first term in the equation is a power law decay (Porod-like scattering) which describes the scattering from clusters or aggregates in the system. The second term is a Lorentzian function that corresponds to the scattering of individual chains in solution (association contribution), where $\xi$ is the key parameter. 
Physicochemical stability study. The physicochemical stability of the NLC formulations was monitored $(\mathrm{n}=3)$ for 12 months at $25 \pm 2{ }^{\circ} \mathrm{C}$ and $60 \pm 5 \%$ relative humidity (ICH 2009). The evaluated parameters were nanoparticle size $(\mathrm{nm})$, PDI and $\mathrm{ZP}(\mathrm{mV})$, plus visual analysis. Analysis of variance $($ ANOVA, $\mathrm{p}<0.05)$ and Tukey's post-hoc tests were used to compare significant differences over time.

Lipid miscibility assessed by Raman mapping. The samples were prepared by heating the solid lipids (CP, MM) $10^{\circ} \mathrm{C}$ above their melting temperatures, followed by addition of the liquid lipid (TRANS, DK) under stirring until a visually homogeneous mixture was obtained. The concentration of the liquid lipid was set to $30 \%(\mathrm{w} / \mathrm{w})$ for the analyses. The samples were cooled to room temperature in an aluminum cell and an area of $1.95 \times 1.95 \mathrm{~mm}^{2}$ was mapped in a Raman Station 400 (Perkin Elmer, Waltham, USA) using a laser of $785 \mathrm{~nm}$ as an excitation light and nominal power of $100 \mathrm{~mW}$. The exposure time was set at $3 \mathrm{~s} / \mathrm{pixel}, 2$ exposures/pixel, $50 \mu \mathrm{m}$ pixel size, in the spectral range of $3200-600 \mathrm{~cm}^{-1}$, with $4 \mathrm{~cm}^{-1}$ resolution. Each sample provided a data cube whose dimension was $40 \times 40 \times 651$, where 40 represents the number of pixels at $x$ and $y$ axis, and 651 is the number of spectral variable/Raman shift.

Chemometric analysis. Spikes on Raman spectra were excluded using an algorithm written in Matlab ${ }^{25}$. After exclusion of spikes, the data cube was unfolded to a 2D matrix, where pixel position on axis $x$ and $y$ were rows, and spectral variables were the columns. Thus, each sample provided a matrix of 1600 spectra $\times 651$ variables (Raman shifts). The spectra were smoothed using Savitzky-Golay (width $=5$, order of polynomial =2), with baseline correction by weighted least squares and normalization by unit vector. The spectral range was 1804$724 \mathrm{~cm}^{-1}$ for CP-TRANS and MM-DK, and $2964-844 \mathrm{~cm}^{-1}$ for CP-DK samples. The classical least squares (CLS) method was used to generate the maps of concentration (chemical images). CLS considers that the spectrum of a mixture is the sum of the spectra of the pure compounds weighted by their concentrations ${ }^{16,26}$. The standard deviation of the histograms ( $\left.\mathrm{SD}_{\text {hist }}\right)$ of these maps was used to assess the miscibility between SL and LL. Data analyses were performed using Matlab R2013b (Mathworks Inc., Natick MA, US) and PLS Toolbox version 8.2 (Eigenvector Research Inc., Wenatchee WA, US).

In vitro release experiments. The in vitro release of TTC, free (in solution) or encapsulated by NLC (CPTRANS/TTC, CP-DK/TTC, MM-DK/TTC), was measured using a Franz vertical diffusion cell system under sink condition ${ }^{27}$. The test samples $(0.4 \mathrm{~mL})$ were placed in the donor compartment of the diffusion cells, which was separated by a polycarbonate membrane (Nucleopore Track-Etch, $0.1 \mathrm{~mm}$ pore size, Whatman) ${ }^{28}$ from the acceptor compartment containing $4 \mathrm{~mL}$ of the release medium ( $5 \mathrm{mM} \mathrm{PBS,} \mathrm{pH} 7.4$ with 5\% Tween 80). The system was kept at $37{ }^{\circ} \mathrm{C}$ under magnetic stirring $(300 \mathrm{rpm})$. At predetermined intervals during $50 \mathrm{~h}$, aliquots $(0.2 \mathrm{~mL})$ were extracted from the acceptor compartment and the volume was replaced with the release medium. The concentration of released TTC in the aliquots was determined by HPLC $(n=5)$.

The release curves were analyzed with the KinetDS 3.0 software ${ }^{29}$. Several kinetic models were tested and according to the coefficient of determination $\left(\mathrm{R}^{2}\right)$ the best fit for NLC formulation curves was reached with the Korsmeyer-Peppas model:

$$
Q=k \cdot t^{n}
$$

where $Q$ is fraction of drug released at time $t, k$ is the rate constant, and $n$ is the release exponent that typifies the drug release mechanism: $n=0.43$ indicates Fickian diffusion, $n=1$ means zero-order release while $0.43<n<1$ values are related to anomalous transport ${ }^{30}$.

Cell viability tests. Balb/c 3T3 murine fibroblasts were used and cell viability was measured by reduction of MTT. Cells were cultured in RPMI 1640 medium supplemented with $10 \%$ fetal bovine serum and $1 \%$ antibiotic (penicillin and streptomycin). Cells $\left(1.0 \times 10^{4}\right.$ cells $\left./ \mathrm{mL}^{-1}\right)$ in RPMI medium and incubated in 96-well plates for $24 \mathrm{~h}$ at $37{ }^{\circ} \mathrm{C}$ under humidified atmosphere and $5 \% \mathrm{CO}_{2}$ were treated with different concentrations of the samples (free TTC, CP-TRANS/TTC, CP-DK/TTC, MM-DK/TTC) diluted in the medium, for $24 \mathrm{~h}$. The treatment medium was removed, the plates washed with sterile PBS buffer $\mathrm{pH} 7.4$, and $100 \mu \mathrm{L}$ of MTT solution $\left(0.5 \mathrm{mg} \mathrm{mL}^{-1}\right.$ in culture medium) was added to each well. After incubation for $3 \mathrm{~h}$ at $37^{\circ} \mathrm{C}$, the MTT solution was removed, and the formed formazan crystals were solubilized in $100 \mu \mathrm{L}$ of ethanol. The plates were shaken for $5 \mathrm{~min}$, and the absorbance of each well was read at $570 \mathrm{~nm}$. Values were expressed as percent MTT reduction, in comparison to control (untreated cells). The analyses were performed in triplicate, and the results expressed as mean \pm SD. The statistical analysis of the results was performed by One-way ANOVA, followed by Tukey's test $(\mathrm{p}<0.05)$.

\section{Results and discussion}

This study reports the development of three optimized NLC pre-formulations capable of encapsulating TTC at high doses (4\%). The NLC were prepared with mixtures of solid and liquid lipids (CP-TRANS, CP-DK and MM-DK), plus P68 as a stabilizer. The choice of lipids and their concentrations was based on previous reports ${ }^{8,16}$. Differently than a previous report in the literature in which TTC was incorporated in NLC aimed for topical application $^{31}$ the formulation in here is to be used by different routes of administration.

Experimental design. In order to analyze the factors that influence the different NLC formulations, three $2^{3}$ factorial designs with triplicate at the central point were carried out. Using three independent variables: (a) surfactant concentration (\% P68), (b) total lipid concentration (\% TL), and (c) solid:liquid lipid ratio (expressed 

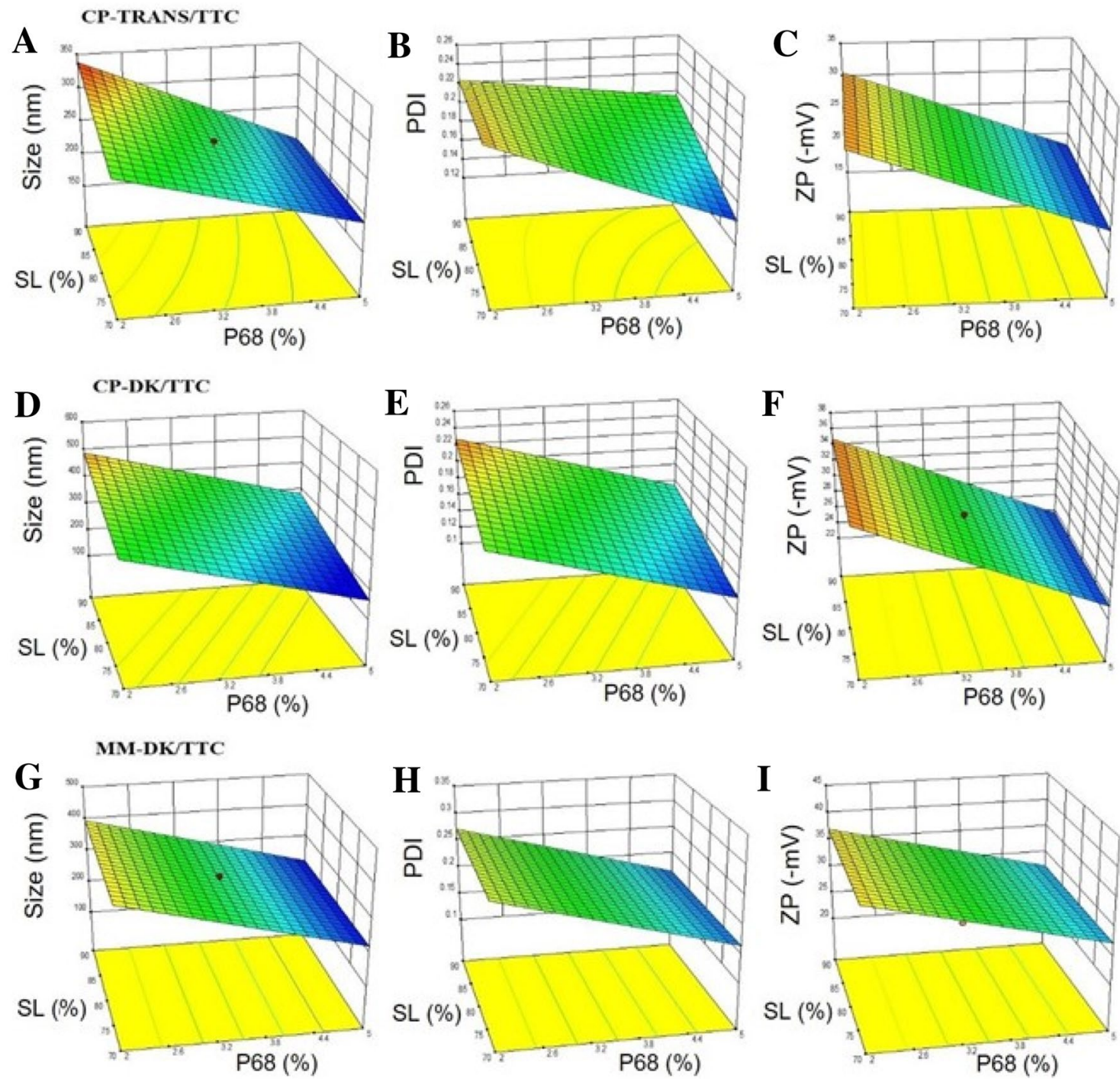

Figure 1. Response surfaces of the three different NLC formulations (CP-TRANS/TTC, CP-DK/TTC, MM-DK/TTC), at $18 \%$ total lipid concentration, regarding: size (A, D, G), PDI (B, E, H), and ZP (C, F, I).

as \% SL), eleven NLC formulations (of nine different compositions) containing 4\% TTC were prepared in each of the $2^{3}$ factorial design. Table S1 shows the composition of these systems and corresponding experimental responses (size, PDI, ZP), determined by DLS.

The mean particle size of the NLC ranged from 250 to $330 \mathrm{~nm}$. Table S2 shows that in the formulations composed of CP-TRANS/TTC, two variables significantly affected the particle size: \% P68, \% SL and their interactions $(\mathrm{p}<0.05)$, as exemplified in Fig. 1A. For CP-DK/TTC and MM-DK/TTC only \% P68 (Fig. 1D,G and Table S2) significantly influenced particle size. The positive effect of the amount of solid lipid (\% SL) on the size of CPTRANS/TTC nanoparticles is a consequence of the higher viscosity of the lipid phase, reducing the effectiveness of particle breaking (homogenization and sonication) processes ${ }^{32,33}$ and will also be discussed further (SANS and Raman Imaging data). For all the 3 NLC types the surfactant concentration (\% P68) had a negative effect on the particle size, which means that the increase in the concentration of P68 decreases the particle size, due to the decrease of interfacial tension between the nanoparticles and the external phase ${ }^{33}$.

The low PDI values $(0.1>$ PDI $>0.25)$ in Table S1 confirmed the homogenous distribution of particle sizes for CP-TRANS/TTC, CP-DK/TTC and MM-DK/TTC. Figure 1B,E,H and Table S2 revealed that only P68 had a significant (negative) effect, decreasing PDI in the 3 NLC formulations, a result that confirms that surfactants play a major role in determining the size distribution of the nanoparticles.

$\mathrm{ZP}$ values in the three types of formulations were in the range of -20 to $-40 \mathrm{mV}$ (Table S1) and P68 was the only variable that significantly affected $\mathrm{ZP}$ values in a negative way: the higher the P68 concentration the lower, in modulus, the surface electric potential of the NLC (Table S2, Fig. 1C,F,I). The negative ZP values are attributable to the polarization of surfactant P68, followed by adsorption of water molecules on the polarized surfaces of $\mathrm{NLC}^{32-37}$. 


\begin{tabular}{|l|l|l|l|r|l|l|}
\hline Formulation & Size $(\mathbf{n m})$ & PDI & ZP $(\mathbf{m V})$ & NC $\left(\times \mathbf{1 0}^{\mathbf{1 3}} / \mathbf{m L}\right)$ & \%EE & \%DL \\
\hline CP-TRANS & $166.6 \pm 1.4$ & $0.115 \pm 0.031$ & $-17.4 \pm 0.6$ & $7.81 \pm 0.43$ & - & \\
\hline CP-TRANS/TTC & $199.0 \pm 2.5$ & $0.187 \pm 0.028$ & $-21.8 \pm 1.5$ & $7.77 \pm 0.19$ & $68.1 \pm 3.5$ & $11.1 \pm 0.2$ \\
\hline CP-DK & $189.9 \pm 2.9$ & $0.112 \pm 0.015$ & $-19.0 \pm 0.9$ & $11.50 \pm 0.34$ & - & \\
\hline CP-DK/TTC & $215.8 \pm 1.8$ & $0.172 \pm 0.010$ & $-27.8 \pm 0.5$ & $9.20 \pm 0.98$ & $65.0 \pm 2.5$ & $11.6 \pm 0.4$ \\
\hline MM-DK & $193.3 \pm 2.7$ & $0.139 \pm 0.021$ & $-29.0 \pm 1.7$ & $10.80 \pm 0.33$ & - & \\
\hline MM-DK/TTC & $222.2 \pm 2.6$ & $0.154 \pm 0.020$ & $-30.1 \pm 0.3$ & $6.77 \pm 0.48$ & $63.7 \pm 4.2$ & $11.6 \pm 0.8$ \\
\hline
\end{tabular}

Table 2. Particle size, polydispersity (PDI), zeta potential (ZP), nanoparticle concentration (NC), encapsulation efficiency (\%EE) and drug loading capacity (\%DL) of the optimized NLC formulations $($ mean $\pm \mathrm{SD}, \mathrm{n}=3$ ).

Desirability functions were used to determine the preferred formulation, following the criteria: lower particle sizes, minimum PDI and maximum ZP values. According to that, the following systems were selected: CP-TRANS/TTC $=20 \%$ TL, 70:30\% SL, and 4.4\% P68, CP-DK/TTC $=18 \%$ TL, 70:30\% SL, and 4.4\% P68 and MM-DK/TTC $=17 \%$ TL, 72:28\% SL, and 5\% P68. The subsequent experiments were conducted only with these optimized formulations.

DLS and NTA results. Table 2 shows the average size, PDI, ZP and nanoparticle concentration (NC) values for the optimized formulations revealed by Factorial Design, and their respective controls. The three types of NLC formulations displayed sub-micron diameters $(\sim 200 \mathrm{~nm})$ and monodisperse size distribution $(0.1<\mathrm{PDI}<0.20)$ with proper electrical charge repulsion between the particles $(\mathrm{ZP}>|17| \mathrm{mV})$ to ensure good shelf-stability ${ }^{36-40}$. Visual analysis confirmed the homogeneous appearance of the fresh samples, with suspensions of whitish coloring, liquid consistency and no evidence of aggregates.

NTA is an alternative methodology to DLS for in vitro characterization of nanostructured colloidal systems ${ }^{9}$. NTA measurements allowed determination of the nanoparticles concentration (NC) in the optimized formulations (Table 2), an analytical parameter used in nanotoxicity, pharmacokinetic and stability (rupture, aggregation) studies of nanoparticles ${ }^{41,42}$. The slightly lower NC values of CP-TRANS/TTC, CP-DK/TTC, MM-DK/TTC reflect their higher sizes, in comparison to the controls without tetracaine.

Most importantly, we have used the NC values and \%EE to estimate the number of molecules of each excipient per nanoparticle ${ }^{8}$, as shown in Table S3. These numbers revealed a significant number of TTC molecules inside each particle $\left(6-8 \times 10^{5}\right)$, corresponding to TTC:total lipid molar ratios of $0.14-0.17$ that justify the increased diameter of TTC-containing nanoparticles.

Tetracaine encapsulation efficiency and drug loading. The three types of NLC formulations developed in this study showed very good capacity to carry TTC, with \%EE values ranging from 63.7 to $68.1 \%$ and $\% \mathrm{DL}>11$ (Table 2), reflecting the strong partition of the non-ionized form of TTC in the lipid milieu ${ }^{43}$. Indeed, the encapsulation efficiency determined for TTC in these nanoparticles was in the range of those reported $(>55 \%)$ for other hydrophobic local anesthetics such as dibucaine, bupivacaine and ropivacaine ${ }^{16,44,45}$ and higher $(<37 \%)$ than those observed in NLC with more hydrophilic agents such as lidocaine and prilocaine ${ }^{8}$. The drug loading capacity is another parameter that expresses the upload capacity of DDS (Eq. 2). The DL values of the optimized formulations for TTC were $11.1 \%$ with CP-TRANS/TTC and $11.6 \%$ with CP-DK/TTC and MM-DK/ TTC, above those reported $(\% \mathrm{DL}<10)$ for other local anesthetics in $\mathrm{NLC}^{16,46,47}$. \%DL values were also in good agreement with the TTC:TL ratios (Table S3).

Transmission electron microscopy. TEM images showed that the nanoparticles, in despite of the different lipid matrices, had spherical morphology with a well-delimited surface (Fig. 2). In addition, encapsulation of TTC did not affect the integrity of the nanoparticles (Fig. 2A,C,E vs. B,D,F).

Infrared analysis (ATR-FTIR). ATR-FTIR analyses were performed to investigate possible interactions between the anesthetic and the lipid matrix of NLC. Figure 3A shows the spectra of NLC excipients and TTC, while the spectra of the optimized NLC formulations and their controls are given in Fig. 3B. TTC spectrum showed absorption bands at 3370 and $1532 \mathrm{~cm}^{-1}$ attributed to $\mathrm{N}-\mathrm{H}$ groups and $\mathrm{C}-\mathrm{N}$ stretching vibrations from the aromatic amine group, respectively, while the bands at 2952 and $2861 \mathrm{~cm}^{-1}$ are due to asymmetric $\mathrm{CH}_{3}$ stretching and symmetrical $\mathrm{CH}_{2}$ stretching vibrations, respectively. Other bands at $1683 \mathrm{~cm}^{-1}$ corresponding to the $\mathrm{C}=\mathrm{O}$ stretching vibration of ester and $1600 \mathrm{~cm}^{-1}$ due to $\mathrm{C}=\mathrm{C}$ of the aromatic ring were detected, as well as those at 1168 and $1118 \mathrm{~cm}^{-1}$ which refer to antisymmetric and symmetric stretching of $\mathrm{C}-\mathrm{O}-\mathrm{C}$, respectively $\mathrm{y}^{5,48}$.

Control NLC (CP-TRANS, CP-DK and MM-DK) showed bands related to their major components (the solid lipids CP and MM) at $2917 \mathrm{~cm}^{-1}$ and $2849-2850 \mathrm{~cm}^{-1}$ corresponding, respectively, to $v_{\mathrm{a}} \mathrm{C}-\mathrm{H}$ and $v_{\mathrm{s}} \mathrm{C}-\mathrm{H}$ vibration modes of $\mathrm{CH}_{2}$. Other bands related to ester bonds were observed in $1733 \mathrm{~cm}^{-1}(\nu \mathrm{C}=\mathrm{O}) ; 1463 \mathrm{~cm}^{-1}$ and $1342-1343 \mathrm{~cm}^{-1}\left(\delta \mathrm{C}-\mathrm{H} \text { in } \mathrm{CH}_{2}\right)^{15,49}$. Finally, characteristic bands of $\mathrm{P} 68$ molecule were observed at $963 \mathrm{~cm}^{-1}$ to $1108 \mathrm{~cm}^{-1}$ and attributed to the symmetrical structure of $\mathrm{C}-\mathrm{O}$ and the asymmetric stretching vibrations of $\mathrm{C}-\mathrm{O}$ in the ether groups of $-\mathrm{OCH}_{2} \mathrm{CH}_{2}$ residues, repeated throughout the structure of $\mathrm{P}^{5} 8^{50}$.

ATR-FTIR spectra of NLC loading TTC showed similarities to those of the control NLC spectra (without TTC), indicating that incorporation of the anesthetic did not affect the overall arrangement of the NLC excipients 

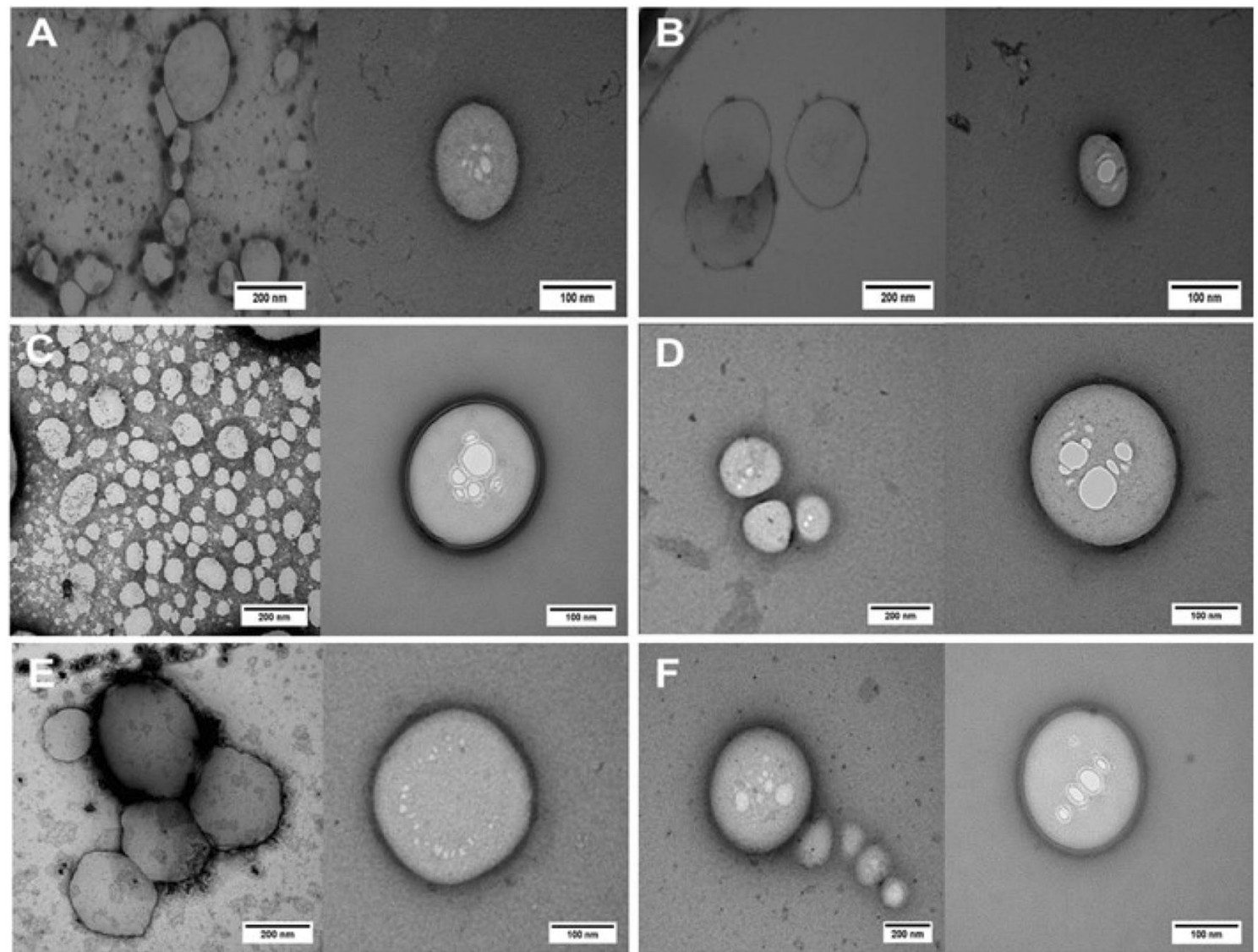

Figure 2. TEM images of NLC formulations and their controls (without TTC): CP-TRANS/TTC (A), CP-TRANS (B), CP-DK/TTC (C), CP-DK (D), MM-DK/TTC (E) and MM-DK. Magnification: $\times 60,000$ (left) (E) $\times 100.000$ (right). Images edited with ImageJ software v.1.52a (https://imagej.nih.gov/ij/).
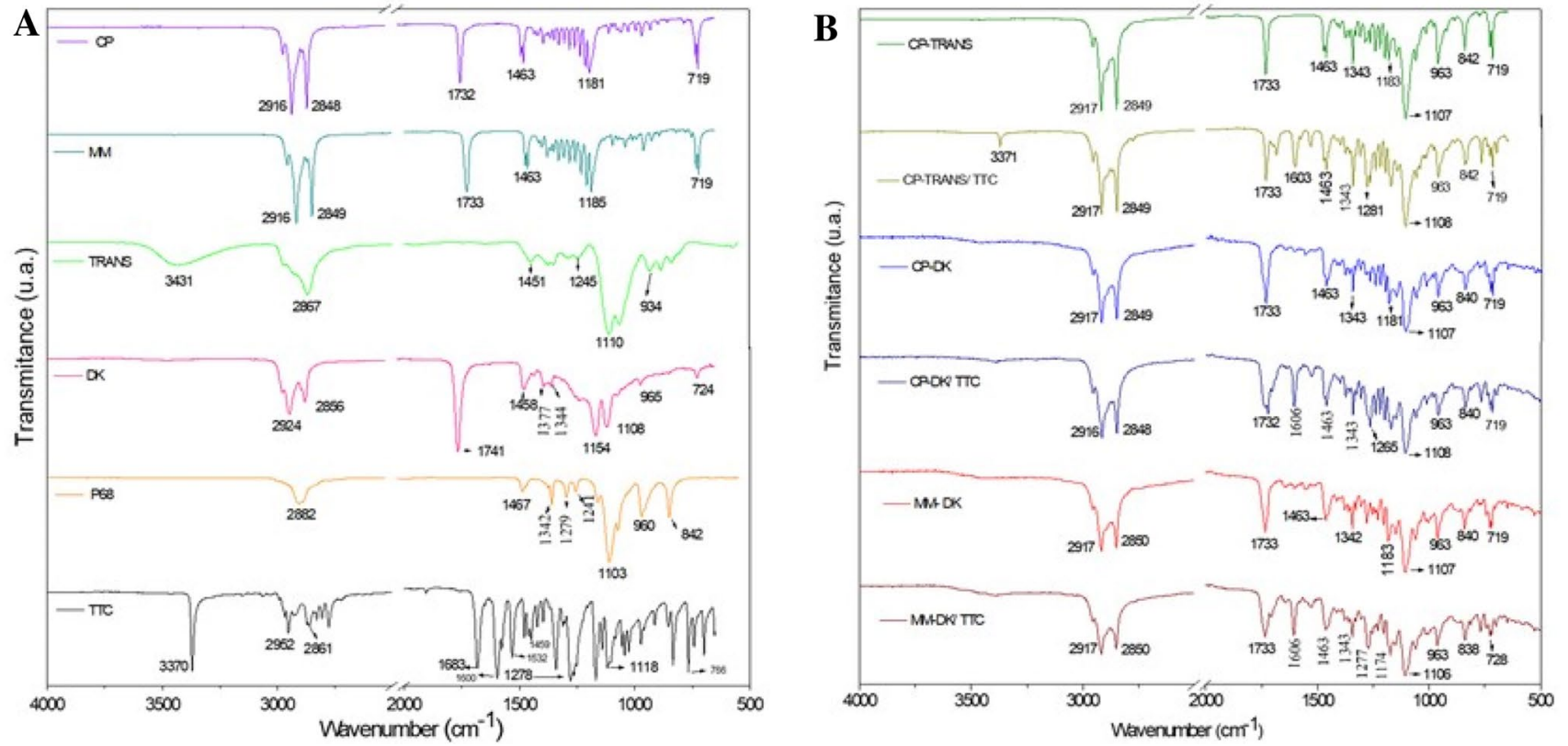

Figure 3. FTIR analysis of TTC, excipients (cetyl palmitate (CP), myristyl myristate (MM), Transcutol (TRANS), Dhaykol (DK), Pluronic F68 (P68), TTC-containing nanoparticles (CP-TRANS/TTC, CP-DK/TTC and MM-DK/TTC) and their controls (CP-TRANS, CP-DK, MM-DK). 

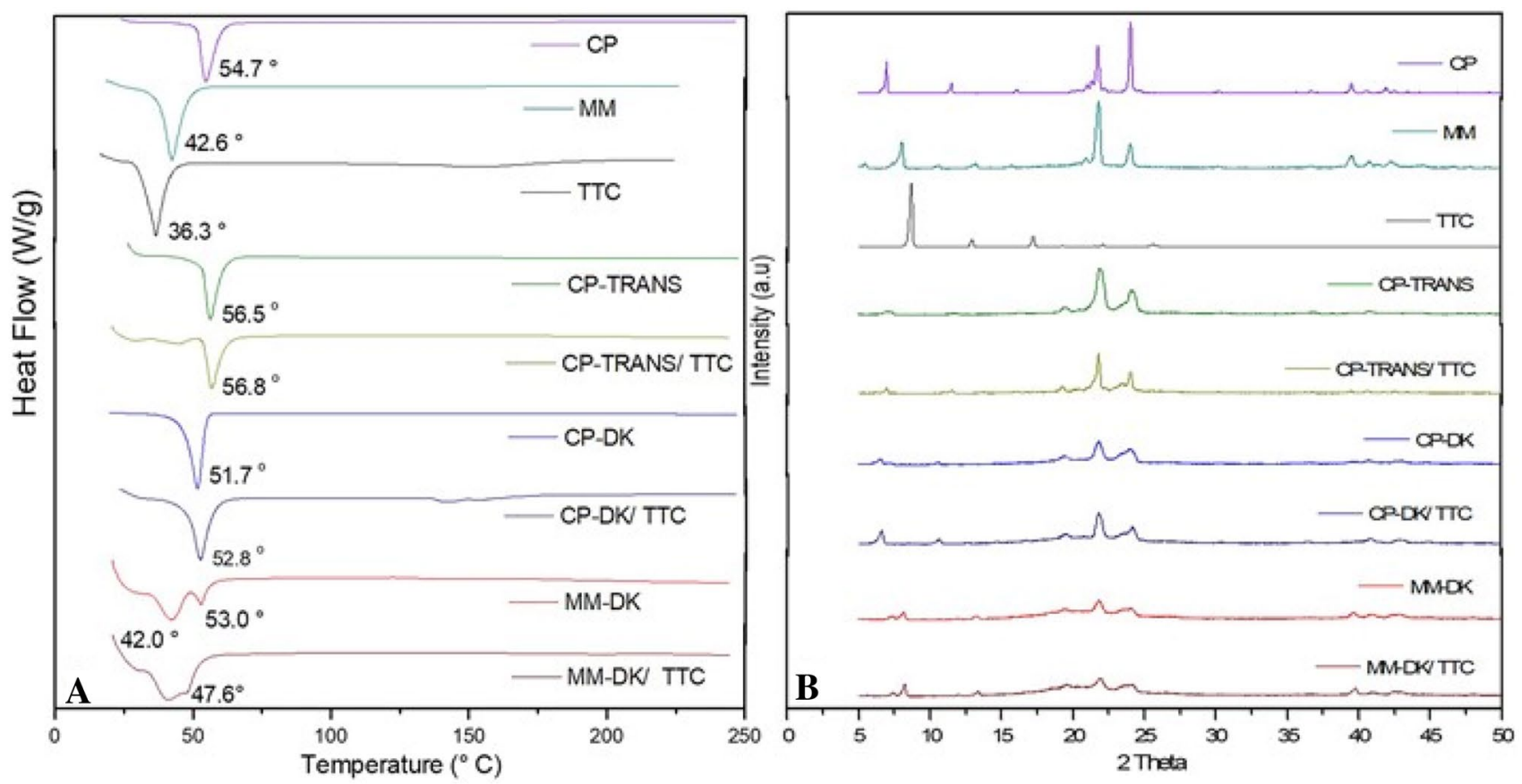

Figure 4. DSC (A) and XRD (B) analyses of nanostructured lipid carriers containing tetracaine (CP-TRANS/ TTC, CP-DK/TTC and MM-DK/TTC), their controls (CP-TRANS, CP-DK and MM-DK), major excipients (CP, MM) and TTC base.

in the nanoparticle $e^{8}$. Among all the NLC spectra, only those of formulations containing tetracaine exhibited typical bands of pure TTC at $1278 \mathrm{~cm}^{-1}$ (C-N stretching) and $1683 \mathrm{~cm}^{-1}(\mathrm{C}=\mathrm{O}$ stretching) which were shifted to $1281-1285 \mathrm{~cm}^{-1}$ and $1603-1606 \mathrm{~cm}^{-1}$, respectively. Such displacements confirm the insertion of TTC into the NLC, probably due to interactions between the amine groups of TTC and available groups of NLC matrices, as described before ${ }^{49}$.

Differential scanning calorimetry (DSC) analysis. Figure 4A shows the thermograms obtained with the optimized NLC formulations, their major (solid lipid) excipients or TTC. The peak belonging to TTC $\left(36.3{ }^{\circ} \mathrm{C}\right)$ could not be seen in any of the three optimized formulations, indicating insertion of the anesthetic inside the nanoparticles ${ }^{51}$. In agreement with the literature, endothermic peaks corresponding to the melting of $\mathrm{CP}$ and $\mathrm{MM}$ were observed at 54.7 and $42.6{ }^{\circ} \mathrm{C}$, respectively ${ }^{15,52}$. Incorporation of the liquid lipids slightly changed the transition of cetyl palmitate to higher (CP-TRANS) or lower temperatures (CP-DK) or, in the case of myristyl myristate, to lower temperatures (MM-DK) plus the appearance of another transition at higher temperature $\left(53^{\circ} \mathrm{C}\right)$. The decrease in the transition of the solid lipid, observed with CP-DK and MM-DK, are expected since the liquid lipid causes a reduction in crystallinity of the solid lipid ${ }^{51}$. As for the shift in the transition of cetyl palmitate to higher temperatures $\left(56.5^{\circ} \mathrm{C}\right.$ in the case of CP-TRANS) it indicates an increase in the crystallinity index of the solid lipid inside the NLC, probably because of miscibility problems with TRANS (as will be discussed latter, in the Raman imaging results). This shift was also observed in the presence of tetracaine (56.8 ${ }^{\circ} \mathrm{C}$ for CP-TRANS/TTC).

X-ray diffraction (XRD) analysis. X-ray diffraction experiments provided information regarding the crystalline structure of NLC and TTC. Figure 4B shows the diffractograms of the optimized NLC formulations, their controls, major excipients (solid lipids, CP and MM) and TTC. The diffractogram of TTC showed three typical peaks at $2 \theta=8.66^{\circ}, 12.90^{\circ}$ and $17.23^{\circ}$, and other peaks of lower intensity, confirming the crystalline nature of the anesthetic ${ }^{53}$. These narrow peaks were not detected in the diffractograms of the optimized formulations, indicating that the anesthetic was solubilized in the lipid matrix of the NLC. In addition, diffractograms of control NLC (CP-TRANS, CP-DK and MM-DK) were different from those of the pure solid lipids (CP and $\mathrm{MM})$, showing peaks of lower intensities $\left(2 \theta=6.94^{\circ}, 21.72^{\circ}, 24.02^{\circ}\right.$ for $\mathrm{CP}$, and $2 \theta=7.96^{\circ}, 21.78^{\circ}, 23.97^{\circ}$ for $\mathrm{MM}$, respectively). These data indicate the lower crystallinity of the NLC lipid matrix in relation to the pure lipids $(\mathrm{CP}, \mathrm{MM})$, thus reflecting a less ordered structure that results from the presence of liquid lipids in the core of the nanoparticles.

The diffraction patterns of TTC-containing particles CP-DK/TTC, MM-DK/TTC and their respective controls (CP-DK and MM-DK) were similar, confirming that addition of TTC did not change the overall organization of these nanoparticles, in agreement with TEM (Fig. 2) and DSC (Fig. 4A) data. In the case of CP-TRANS/ TTC sample there is a narrowing in the more intense CP peaks at $21.72^{\circ}$ and $24.02^{\circ}$ promoted by TTC, indicating that the anesthetic increases the crystallinity of cetyl palmitate, in agreement with DSC results. 

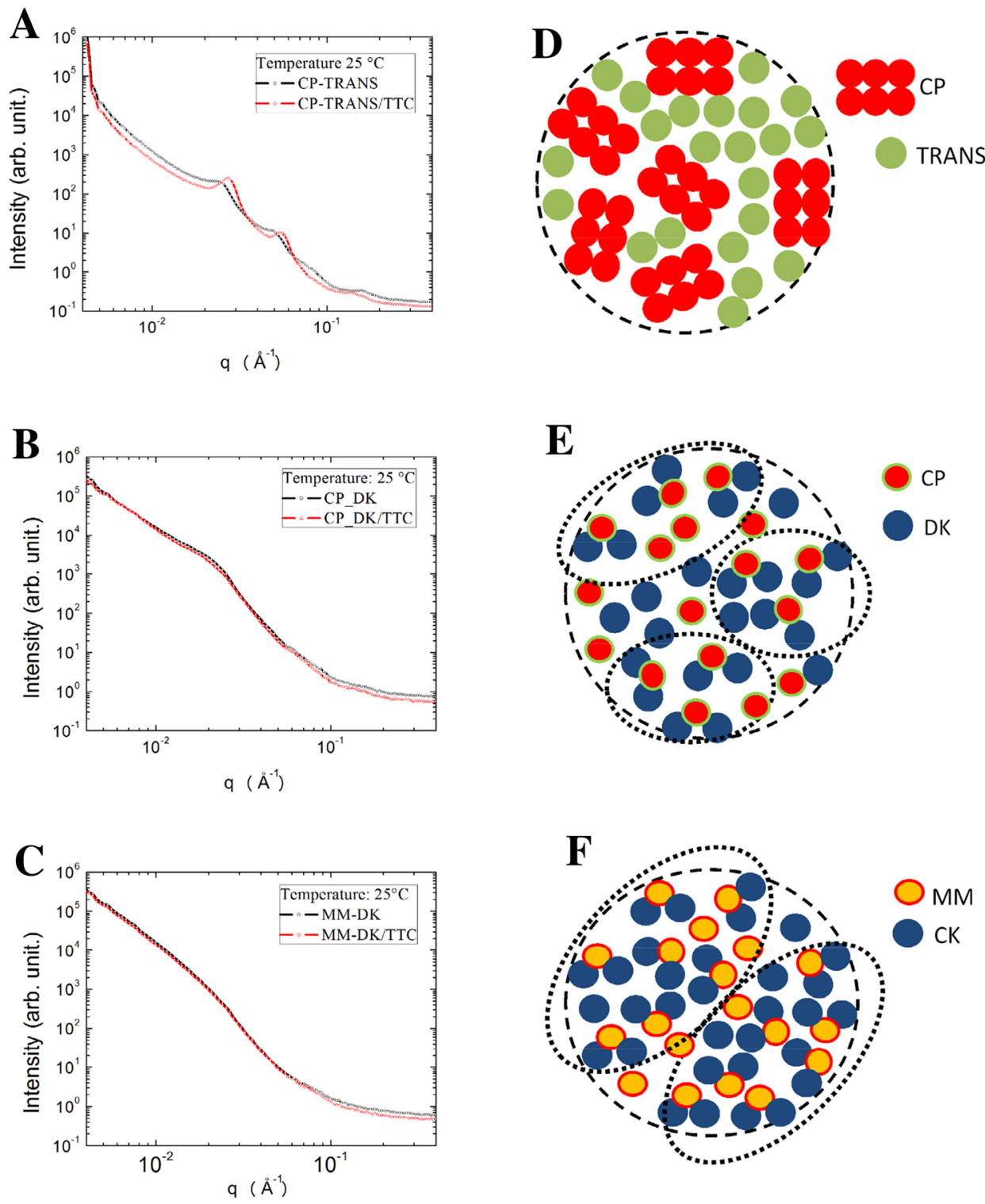

Figure 5. SANS data, measured at $25^{\circ} \mathrm{C}$, for the optimized NLC formulations and their controls (without TTC): (A) CP-TRANS, (B) CP-DK, (C) MM-DK. (D-F) Schematic representation of the lipid arrangements in the inner core of the three kind of nanoparticles (for the sake of clarify the surfactant molecules are not represented). Notice that a lamellar structure was detected in the core of CP-TRANS particles (D) while hydrophobic clusters (dotted lines) were observed between the lipids CP-DK (E) and MM-DK systems (F).

Small angle neutron scattering. SANS measurements were performed in order to get further information on the structural organization of the optimized NLC, with and without tetracaine. The samples were prepared in $\mathrm{D}_{2} \mathrm{O}$ to reach a significant contrast between the solvent and the nanoparticles. First, all the NLC systems exhibit negligible changes when measurements were conducted at $25^{\circ} \mathrm{C}$ and at $37{ }^{\circ} \mathrm{C}$ (as shown in Figure $\mathrm{S} 1$ for CP-DK/TTC and MM-DK/TTC). SANS data then revealed several systematic tendencies in the internal arrangement of the nanoparticles (Fig. 5). For those prepared with cetyl palmitate and Transcutol (CP-TRANS/ TTC, CP-TRANS) correlation peaks in the SANS curves indicated the existence of lamellar structures inside the NLC (Fig. 5A), in agreement with previous reports in the literature, obtained with Electron Paramagnetic Resonance ${ }^{45}$ and molecular Dynamics ${ }^{54}$. Indeed, among the blends of solid and lipid lipids tested, cetyl palmitate and TRANS have the largest difference in polarity, and their SL:LL molar ratio (0.66) was the smallest among the three optimized formulations (Table S3). Because of that, the lamellar structure revealed by SANS results from the reorganization of CP molecules in the lipid NLC core, avoiding the contact with TRANS molecules (see "Discussion" below). Interestingly, and in agreement with that, the Design of Experiments study revealed that only for the CP-TRANS formulation (Fig. 1A) the amount of solid lipid (CP) played a significant effect, determining increased particles size. 
Moreover, inclusion of tetracaine in the CP-TRANS nanoparticles induced a variation in the observed lamellar structure, since the 10 -plane spacing (d10) diminished from $259 \AA$ in CP-TRANS, to $230 \AA$ A in CP-TRANS/ TTC. Such reduction in the lamellar interplanar distances (d10) suggests that tetracaine interacts with the CP molecules, decreasing the thickness or the lamellae by promoting lateral expansion, a phenomenon already observed for TTC in monolayers and bilayers ${ }^{55,56}$. TTC really causes dynamic rearrangements in lamellar phases, as recently demonstrated by $\mathrm{Hu}$ et al. in dioleylphosphatidylcholine supported bilayers, increasing the lipid chain mobility and even inducing the formation of curved tubular structures prior to membrane disruption, at high TTC:lipid ratios $^{57}$.

No such correlation peaks were observed in the SANS profile of the NLC prepared with Dhaykol 6040 as the liquid lipid (Fig. 5B,C). But to gain a further insight about the DK-based NLC systems, we modeled the SANS data using Eq. (3), as shown in Figure S1 ("Supporting information"). The analysis of the correlation length parameter for the CP-DK and MM-DK samples showed that the latter had a bigger correlation length $\left(\xi_{M M-D K}=78.68 \AA\right)$ than the former $\left(\xi_{C P-D K}=44.69 \AA\right)$ suggesting that MM-DK nanoparticles had greater hydrophobic clusters (nanoclusters formed by the hydrophobic interactions between solid and liquid lipids) ${ }^{52}$, in comparison to CP-DK.

In the representations at Fig. 5D-F we depicted the different organizations proposed for the solid and liquid lipids inside the NLC, revealing the hydrophobic clusters observed for CP-DK and MM-DK, but not CP-TRANS. The size of the hydrophobic clusters did not change in the presence of TTC, nor with temperature (Figure S1).

Physicochemical stability studies. A long-term stability study was conducted with the optimized NLC formulations and their controls, by monitoring particle size, PDI, ZP, pH and visual aspects such as color and homogeneity for 365 days at $25^{\circ} \mathrm{C}$. The $\mathrm{pH}$ of the formulations remained in the range of $8.0-8.5$. CP-DK/TTC and MM-DK/TTC and their respective controls did not show any significant variation by visual inspection or in any of the analyzed parameters over time (Fig. 6), reflecting the physical stability of these formulations (i.e. maintenance of nanoparticles structure).

On the other hand, CP-TRANS/TTC and CP-TRANS showed a significant increase in nanoparticle size and polydispersity ( $254.7 \mathrm{~nm}$ and 0.29 , respectively, for CP-TRANS/TTC after 120 days) with ZP values tending to zero $(\mathrm{p}<0.05)$ during storage (Fig. 6$)$. Visual analysis confirmed the instability of this formulation, with phase separation starting after 30 days that prevented analyses after 120 days. Therefore, CP-TRANS NLC was found unstable over time, with changes in particle size, polydispersity and ZP values compatible with particle aggregation $^{37,21}$.

Miscibility of lipid excipients measured by Raman mapping. In an attempt to get more information on the stability of the formulations, Raman imaging analyses were used to evaluate the miscibility of their lipid components. Figure S2 shows Raman spectra of solid and liquid lipids, and their mixtures. These spectra were very similar to prior reports in literature and also previous works from our group ${ }^{16,22}$. As expected, solid lipids had narrower bands than liquid lipids because of their more ordered molecular structures. Assignment of the main bands of each excipient is given in Table S4.

In this case, univariate methods (i.e. single wavenumber) could not be used to treat the data due to the high spectral overlap (Figure S2). Therefore, and since the spectrum of each individual component was available, the multivariate CLS method was employed, allowing the use of all spectra information to generate the chemical images. Figure 7 shows chemical imaging and histograms obtained for the three pairs of solid and liquid lipids of the optimized NLC. Each pair of SL/LL is shown on the left and right sides, respectively of Fig. 7A-C. The predicted mean scores and their ranges for each component in the pixels are given in the histograms. The scores are in the same scale, so they can be directly compared to evaluate lipid miscibility. $\mathrm{SD}_{\text {hist }}$ values of $13.6,3.2$ and 6.1 were found for the lipid mixtures CP-TRANS, CP-DK and MM-DK, respectively. Pixels with 0 or 100 score values would indicate full immiscibility between excipients, but they were not observed in any of the mixtures. CP-TRANS sample showed very wide histograms, with two maxima (Fig. 7A). Such behavior is a clear indication of aggregation, with CP concentrated in the right side (red in the chemical map) and TRANS condensed in the left side (green in the chemical map). The histograms of CP-DK were the narrowest ones (Fig. 7B), and MM-DK showed an intermediate behavior (Fig. 7C). It should be noted that unlike CP-TRANS, the last two systems showed a single distribution, with only one maximum for each (SL and LL) excipient. According to the Raman image analyses, the degree of miscibility in three mixtures decreased in the order: CP-DK $>$ MM-DK $>>$ CPTRANS, corroborating the SANS results (Fig. 5). These results also explain the instability of CP-TRANS under storage, as revealed by DLS (Fig. 6), and confirm the applicability of Raman mapping for the selection of excipients in pharmaceutical studies ${ }^{22,23}$. So, for stability reasons, only the formulations containing DK as the liquid lipid were used in subsequent steps.

In vitro release kinetics. The in vitro release of TTC, in solution and encapsulated in CP-DK/TTC and MM-DK/TTC formulation (Fig. 8), was measured during $48 \mathrm{~h}$ at $37^{\circ} \mathrm{C}$. TTC in solution reached equilibrium (100\% release) in $6 \mathrm{~h}$. The NLC formulations displayed slower TTC release: $40.9 \%$ and $93.0 \%$, respectively for CP-DK/TTC and MM-DK/TTC, after $48 \mathrm{~h}$. The release curves were treated with several kinetic models, and the better fit $\left(r^{2}=0.9991\right.$, and 0.9978 , for CP-DK/TTC and MM-DK/TTC, respectively) was found with the Korsmeyer-Peppas model (Eq. 4). The determined values of $n$ were 0.47 (CP-DK/TTC) and 0.49 (MM+DK/ TTC). According to the Korsmeyer-Peppas model, $n$ values from 0.43 to 1 indicate an anomalous, non-Fickian transpor ${ }^{30}$. This means that probably two mechanisms drove the release of TTC: an initial "burst" release due to the non-encapsulated TTC (ca. 35\%, see Table 2), and a sustained release regimen related to the fraction of TTC loaded by the nanoparticles. The prolonged release of TTC encapsulated in CP-DK/TTC correlates well 

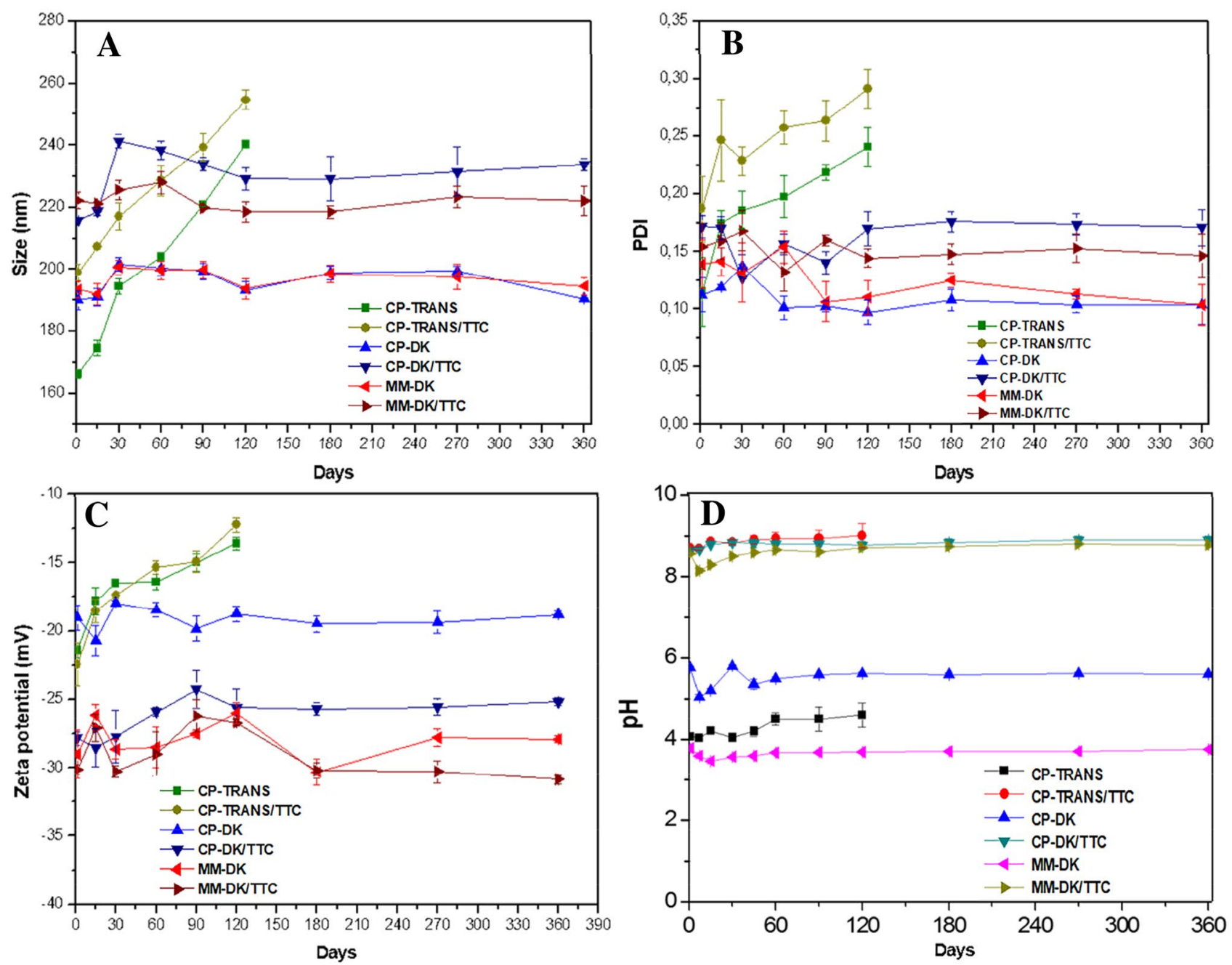

Figure 6. Stability of the optimized formulations during 365 days of storage at $25^{\circ} \mathrm{C}$, considering size (A), PDI (B), $\mathrm{ZP}(\mathbf{C})$ and $\mathrm{pH}(\mathbf{D})$.

with the higher degree of miscibility in the lipid core of these nanoparticles in comparison to MM-CK/TTC, as revealed by Raman Imaging (Fig. 7) and SANS (Fig. 5) data. This modeling confirmed the NLC ability to extend the release of local anesthetics, as previously observed ${ }^{16}$.

In vitro cytotoxicity tests. Finally, we evaluated the cytotoxicity of the optimized (CP-DK/TTC and MM-DK/TTC) formulations through the MTT test (Fig. 9), in cultures of murine Balb/c 3T3 fibroblasts. After $24 \mathrm{~h}$ of treatment, the $\mathrm{IC}_{50}$ of free TTC was $0.6 \mathrm{mM}$, in good agreement with the literature ${ }^{24}$. Slightly higher $\mathrm{IC}_{50}$ values were detected with CP-DK/TTC and MM-DK/TTC formulations ( $0.7 \mathrm{mM}$ and $0.9 \mathrm{mM}$, respectively), showing that the NLC formulations decrease the intrinsic cytotoxicity of TTC, probably due to the sustained drug release over time. Similar observations were reported for TTC encapsulated in NLC composed of glyceryl monostearate, oleic acid and Tween $80^{31}$. Control formulations (CP-DK and MM-DK) were also tested, and they showed no (MM-DK) or low (CP-DK) effect over cell viability at the concentrations tested, indicating the safety of the nanocarrier systems.

\section{Conclusions}

In this study, three NLC pre-formulations for tetracaine (CP-TRANS/TTC, CP-DK/TTC and MM-DK/TTC) were optimized by factorial design and their supramolecular structure was scrutinized with biophysical methods that detected evidence on the interaction of TTC with the lipid core of the NLC. The optimized formulations were capable of encapsulating TTC $(\% \mathrm{EE}>63 \%, \% \mathrm{DL}>11 \%)$ at high doses $(4 \%)$ and the nanoparticles promoted sustained release of TTC beyond $48 \mathrm{~h}$, as evaluated in vitro, at $\mathrm{pH} 7.4$ and $37^{\circ} \mathrm{C}$. But when SANS results revealed details on the lipid core of the NLC, a highly ordered (lamellar) arrangement was observed for CP-TRANS/TTC. In addition, Raman mapping analysis detected the low miscibility between the lipids CP and TRANS, explaining the instability of the CP-TRANS/TTC formulation during storage (Fig. 6), while CP-DK/ TTC and MM-DK/TTC remained stable for 365 days, at $25^{\circ} \mathrm{C}$. The two remaining pre-formulations promoted sustained release and reduced the intrinsic toxicity of TTC over cultured 3T3 cells (MM-DK/TTC > CP-DK/ TTC) in vitro. Therefore, the optimized nanoparticles prepared with DK show interesting properties as carriers 


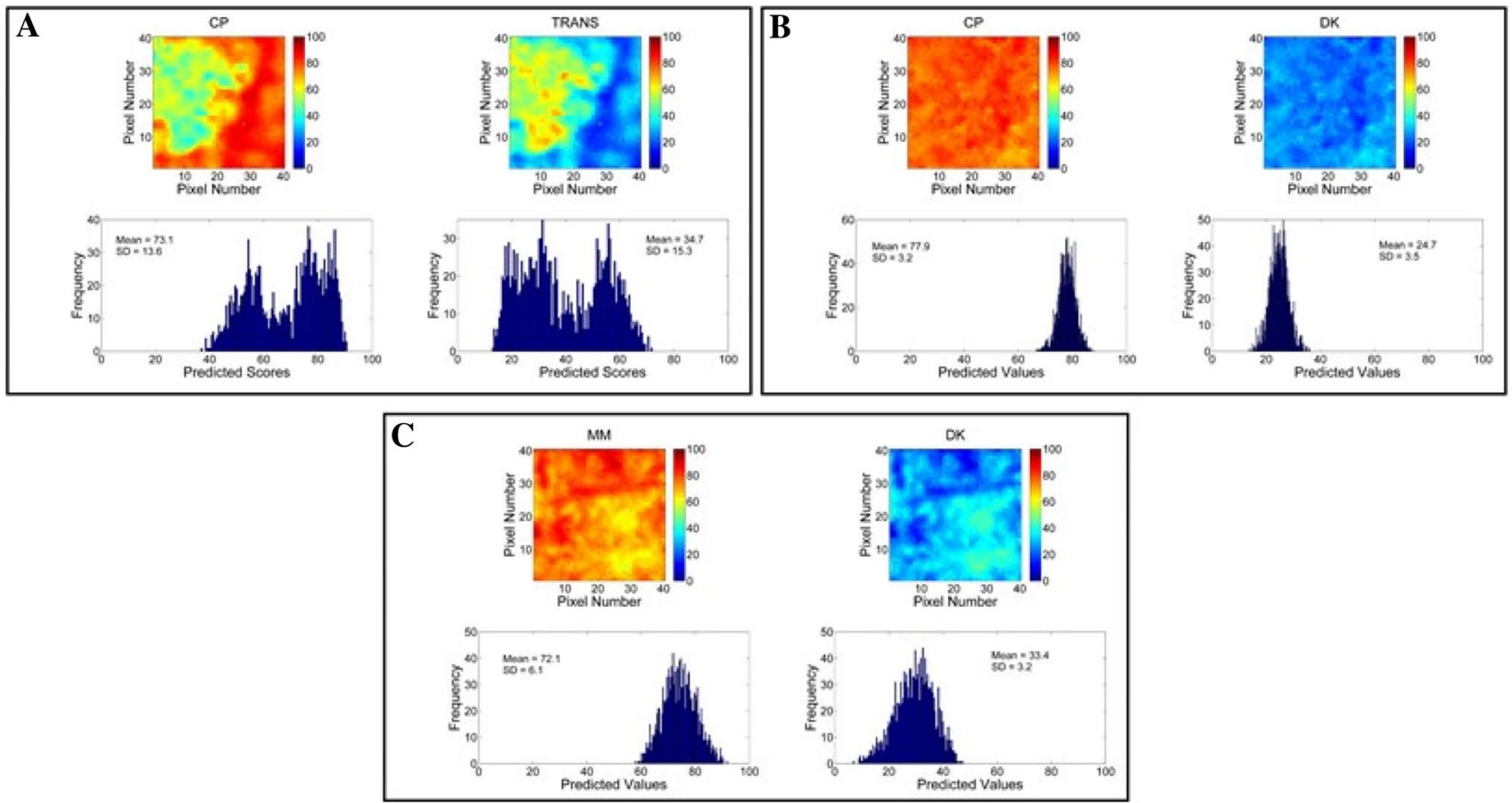

Figure 7. Raman images: distribution maps (top) and histograms (bottom) of predicted values for: (A) CP-TRANS; (B) CP-DK, and (C) MM-DK. For each sample, solid lipids (CP, MM) are represented in the left and liquid lipids (TRANS, DK) in the right.

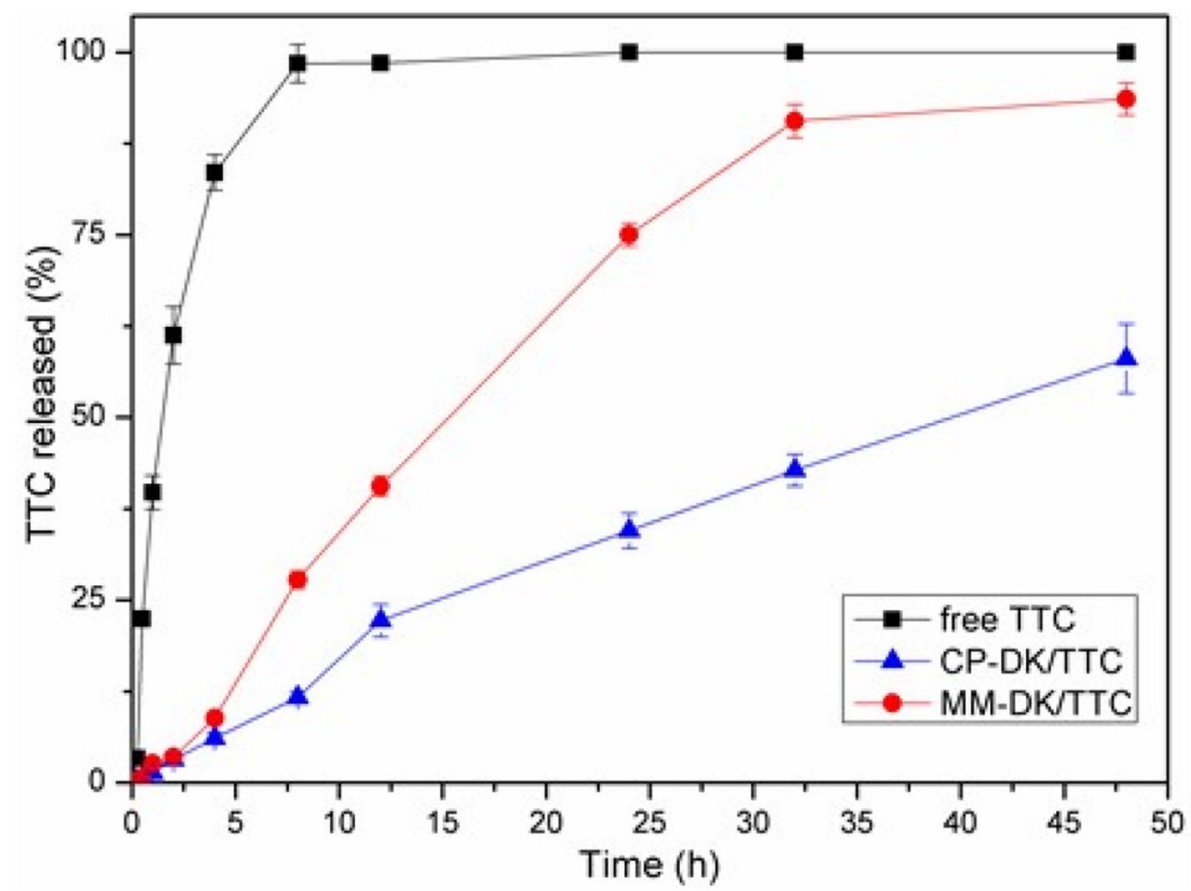

Figure 8. In vitro release profiles of $4 \%$ tetracaine in solution (free TTC) or encapsulated in optimized (CPDK/TTC and MM-DK/TTC) nanoparticles, at $\mathrm{pH} 7.4$ and $37^{\circ} \mathrm{C}(\mathrm{n}=6)$.

for TTC administration, by different routes. Further in vivo studies are necessary to evaluate the therapeutic effect of the optimized pre-formulations and possible reduction of the systemic toxicity of TTC, in comparison to commercially available TTC products. 


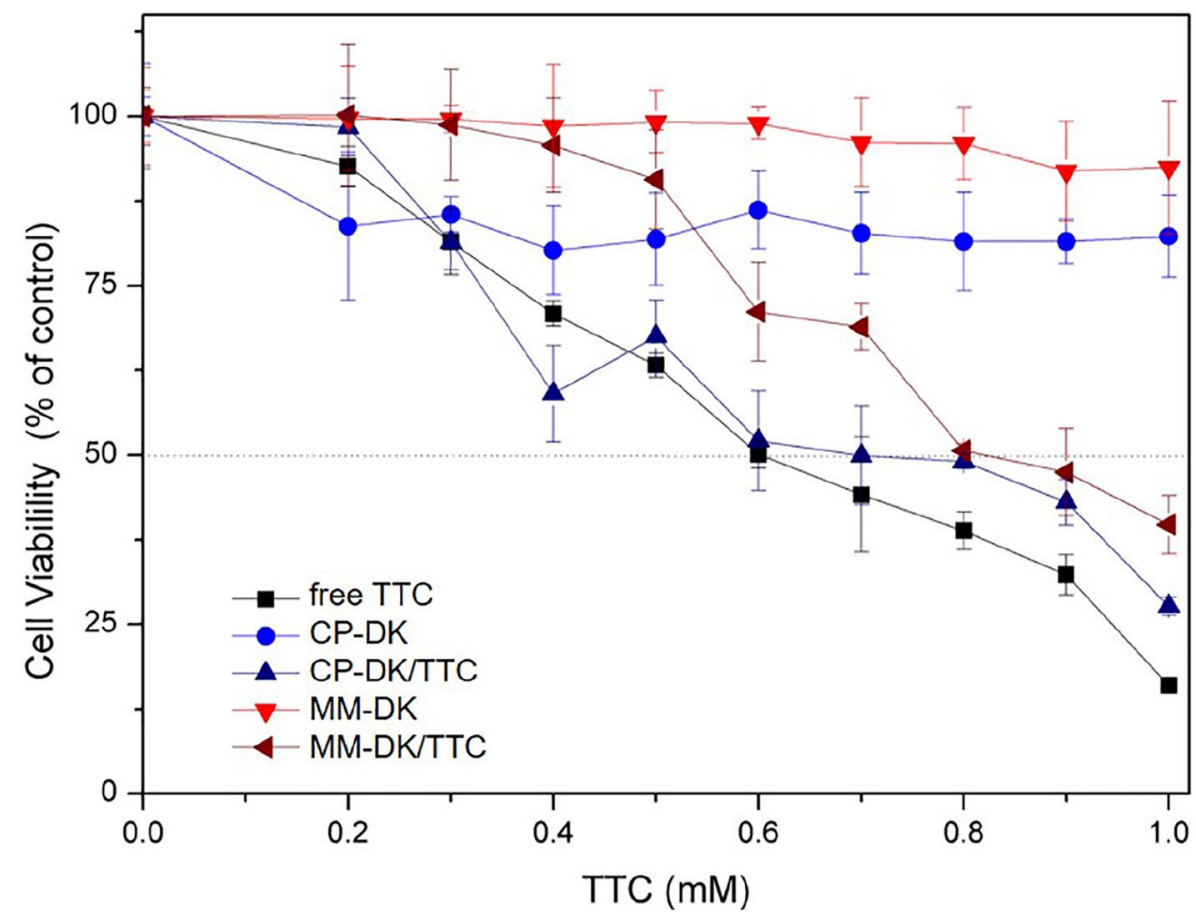

Figure 9. Cytotoxicity evaluation - trough the MTT test-on Balb/c 3T3 fibroblasts treated for $24 \mathrm{~h}$ with TTC in solution (free TTC) or encapsulated in the nanoformulations (CP-DK/TTC, MM-DK/TTC), and their controls (CP-DK, MM-DK), $\mathrm{n}=3$.

Received: 23 March 2021; Accepted: 30 September 2021

Published online: 02 November 2021

\section{References}

1. Fozzard, H. A., Lee, P. J. \& Lipkind, G. M. Mechanism of local anesthetic drug action on voltage-gated sodium channels. Curr. Pharm. Des. 11, 2671-2686 (2005).

2. McLure, H. A. \& Rubin, A. P. Review of local anaesthetic agents. Minerva Anestesiol. 71, 59-74 (2005).

3. Adeleye, A., Sharp, L. \& Rech, M. A. Neurotoxicity secondary to local tetracaine use. Am. J. Emerg. Med. 38(1984), e1-1984.e3 (2020).

4. Teixeira, R. S. et al. Effect of cyclodextrins and $\mathrm{pH}$ on the permeation of tetracaine: Supramolecular assemblies and release behavior. Int. J. Pharm. 466, 349-358 (2014).

5. Inacio, R., Barlow, D., Kong, X., Keeble, J. \& Jones, S. A. Investigating how the attributes of self-associated drug complexes influence the passive transport of molecules through biological membranes. Eur. J. Pharm. Biopharm. 102, 214-222 (2016).

6. de Paula, E. et al. Micro and nanosystems for delivering local anesthetics. Expert Opin. Drug Deliv. 9, 1505-1524 (2012).

7. Silva, G. H. R. et al. Bupivacaine (S75:R25) loaded in Nanostructured lipid carriers: Factorial design, HPLC quantification method and physicochemical stability study. Curr. Drug Deliv. 14, 1-9 (2017).

8. Ribeiro, L. N. M. et al. Nanostructured lipid carriers as robust systems for topical lidocaine-prilocaine release in dentistry. Eur. J. Pharm. Sci. 93, 192-202 (2016).

9. Ribeiro, L. N. M. et al. Natural lipids-based NLC containing lidocaine: From pre-formulation to in vivo studies. Eur. J. Pharm. Sci. 106, 102-112 (2017).

10. de Araújo, D. R., Ribeiro, L. N. M. \& de Paula, E. Lipid-based carriers for the delivery of local anesthetics. Expert Opin. Drug Deliv. 16, 701-714 (2019).

11. Uebbing, L. et al. Investigation of $\mathrm{pH}$-responsiveness inside lipid nanoparticles for parenteral mRNA application using small-angle X-ray scattering. Langmuir 36, 13331-13341 (2020).

12. Fang, C.-L., Al-Suwayeh, S. A. \& Fang, J.-Y. Nanostructured lipid carriers (NLCs) for drug delivery and targeting. Recent Pat. Nanotechnol. 7, 41-55 (2013).

13. Terada, T. et al. Characterization of lipid nanoparticles containing ionizable cationic lipids using design-of-experiments approach. Langmuir https://doi.org/10.1021/acs.langmuir.0c03039 (2021).

14. Schwarz, C., Mehnert, W., Lucks, J. S. \& Muller, R. H. Solid lipid nanoparticles (SLN) for controlled drug delivery. I. Production, characterization and sterilization. J. Control. Release 30, 83-96 (1994).

15. Islan, G. A., Tornello, P. C., Abraham, G. A., Duran, N. \& Castro, G. R. Smart lipid nanoparticles containing levofloxacin and DNase for lung delivery. Design and characterization. Colloids Surfaces B Biointerfaces 143, 168-176 (2016).

16. Rodrigues da Silva, G. H. et al. Optimised NLC: A nanotechnological approach to improve the anaesthetic effect of bupivacaine. Int. J. Pharm. 529, 253-263 (2017).

17. Vogtt, K. et al. A new time-of-flight small-angle scattering instrument at the Helmholtz-Zentrum Berlin: V16/VSANS J. Appl. Cryst.47, 237-244 (2014).

18. Asadujjaman, A., Ahmadi, V., Yalcin, M., Brummelhuis, Bertin, A. Thermoresponsive functional polymers based on 2,6-diaminopyridine motif with tunable UCST behaviour in water/alcohol mixtures. Bertin, Polym. Chem. 8, 3140-3153 (2017).

19. Hammouda, B., Ho, D. Kline, S. SANS from Poly(ethylene oxide)/Water Systems. Macromol. 35, 8578-8585 (2002). 
20. Hammouda, B. SANS from Polymers-Review of the Recent Literature. Polymer Reviews, 50, 14-39 (2020). ICH 2009. International Conference on Harmonisation; guidance on Q10 Pharmaceutical Quality System;availability. Notice. Food and Drug Administration, Fed Regist. 74, 15990-15991 (2009).

21. Ferreira, M., Chaves, L. L., Lima, S. C. \& Reis, S. Optimization of nanostructured lipid carriers loaded with methotrexate: A tool for inflammatory and cancer therapy. Int. J. Pharm. 492, 65-72 (2015).

22. Mitsutake, H. et al. Comparison of different chemometric methods to extract chemical and physical information from Raman images of homogeneous and heterogeneous semi-solid pharmaceutical formulations. Int. J. Pharm. 552, 119-129 (2018).

23. Mitsutake, H. et al. Evaluation of miscibility and polymorphism of synthetic and natural lipids for nanostructured lipid carrier (NLC) formulations by Raman mapping and multivariate curve resolution (MCR). Eur. J. Pharm. Sci. 135, 51-59 (2019).

24. Lima, R. A. F. et al. Improvement of tetracaine antinociceptive effect by inclusion in cyclodextrins. J. Drug Target. 20, 85-96 (2012).

25. Sabin, G. P., de Souza, A. M., Breitkreitz, M. C. \& Poppi, R. J. Desenvolvimento de um algoritmo para identificação e correção de spikes em espectroscopia raman de imagem. Quim. Nova 35, 612-615 (2012).

26. Amigo, J. M. \& Ravn, C. Direct quantification and distribution assessment of major and minor components in pharmaceutical tablets by NIR-chemical imaging. Eur. J. Pharm. Sci. 37, 76-82 (2009).

27. Gonzalez-Mira, E., Egea, M. A., Souto, E. B., Calpena, A. C. \& García, M. L. Optimizing flurbiprofen-loaded NLC by central composite factorial design for ocular delivery. Nanotechnology 22, 045101 (2011).

28. Tiyaboonchai, W., Tungpradit, W. \& Plianbangchang, P. Formulation and characterization of curcuminoids loaded solid lipid nanoparticles. Int. J. Pharm. 337, 299-306 (2007).

29. Mendyk, A. et al. KinetDS: An open source software for dissolution test data analysis. Dissolution Technol. 6, 11. https://doi.org/ 10.14227/DT190112P6 (2012).

30. Ritger, P. L. \& Peppas, N. A. A simple equation for description of solute release. I. Fickian and non-fickian release from nonswellable devices in the form of slabs, spheres, cylinders or discs. J. Control. Release 5, 23-36 (1987).

31. Liu, X. \& Zhao, Q. Long-term anesthetic analgesic effects: Comparison of tetracaine loaded polymeric nanoparticles, solid lipid nanoparticles, and nanostructured lipid carriers in vitro and in vivo. Biomed. Pharmacother. 117, 109057 (2019).

32. Fangueiro, J. F. et al. Experimental factorial design applied to mucoadhesive lipid nanoparticles via multiple emulsion process. Colloids Surfaces B Biointerfaces 100, 84-89 (2012).

33. Pradhan, M., Singh, D., Murthy, S. N. \& Singh, M. R. Design, characterization and skin permeating potential of Fluocinolone acetonide loaded nanostructured lipid carriers for topical treatment of psoriasis. Steroids 101, 56-63 (2015).

34. Zhao, S. et al. Mixture of nonionic/ionic surfactants for the formulation of nanostructured lipid carriers: Effects on physical properties. Langmuir 30, 6920-6928 (2014).

35. Lasoń, E., Sikora, E. \& Ogonowski, J. Influence of process parameters on properties of nanostructured lipid carriers (NLC) formulation. Acta Biochim. Pol. 60, 773-777 (2013).

36. Jain, N. K. \& Ram, A. Development and characterization of nanostructured lipid carriers of oral hypoglycemic agent: Selection of surfactants. Int. J. Pharm. Sci. Revier Res. 7, 125-130 (2011).

37. Han, F., Li, S., Yin, R., Liu, H. \& Xu, L. Effect of surfactants on the formation and characterization of a new type of colloidal drug delivery system: Nanostructured lipid carriers. Colloids Surfaces A Physicochem. Eng. Asp. 315, 210-216 (2008).

38. Muller, R. H., Radtke, M. \& Wissing, S. A. Nanostructured lipid matrices for improved microencapsulation of drugs. Int. J. Pharm. 242, 121-128 (2002).

39. Tamjidi, F., Shahedi, M., Varshosaz, J. \& Nasirpour, A. Nanostructured lipid carriers (NLC): A potential delivery system for bioactive food molecules. Innov. Food Sci. Emerg. Technol. 19, 29-43 (2013).

40. Das, S., Ng, W. K. \& Tan, R. B. H. Are nanostructured lipid carriers (NLCs) better than solid lipid nanoparticles (SLNs): Development, characterizations and comparative evaluations of clotrimazole-loaded SLNs and NLCs?. Eur. J. Pharm. Sci. 47, 139-151 (2012).

41. Mendes, L. P. et al. Biodegradable nanoparticles designed for drug delivery: The number of nanoparticles impacts on cytotoxicity. Toxicol. Vitr. 29, 1268-1274 (2015).

42. Ribeiro, L. N. M., Couto, V. M., Fraceto, L. F. \& de Paula, E. Use of nanoparticle concentration as a tool to understand the structural properties of colloids. Sci. Rep. 8, 982 (2018).

43. de Paula, E. \& Schreier, S. Use of a novel method for determination of partition coefficients to compare the effect of local anesthetics on membrane structure. Biochim. Biophys. Acta 1240, 25-33 (1995).

44. Chen, H. et al. Development of a ropivacaine-loaded nanostructured lipid carrier formulation for transdermal delivery. Colloids Surfaces A Physicochem. Eng. Asp. 465, 130-136 (2015).

45. Barbosa, R. M. et al. Solid lipid nanoparticles for dibucaine sustained release. Pharmaceutics 10, 231 (2018).

46. Nahak, P. et al. Physicochemical studies on local anaesthetic loaded second generation nanolipid carriers. RSC Adv. 5, 26061-26070 (2015).

47. Rodrigues da Silva, G. H. et al. Injectable in situ forming nanogel: A hybrid Alginate-NLC formulation extends bupivacaine anesthetic effect. Mater. Sci. Eng. C 109, 110608 (2020).

48. Velez-Saboyá, C. S., Oropeza-Guzman, E., Sierra-Valdez, F. J. \& Ruiz-Suárez, J. C. Ca ${ }^{2+}$-mediated enhancement of anesthetic diffusion across phospholipid multilamellar systems. Biochim. Biophys. Acta Biomembr. 1863, 183509 (2021).

49. Rodenak-Kladniew, B., Islan, G. A., de Bravo, M. G., Durán, N. \& Castro, G. R. Design, characterization and in vitro evaluation of linalool-loaded solid lipid nanoparticles as potent tool in cancer therapy. Colloids Surfaces B Biointerfaces 154, 123-132 (2017).

50. Seema, M. D. Organoclay Pluronic F68-Montmorillonite, as a sustained release drug delivery vehicle for propranolol hydrochloride. Eur. Chem. Bull. 3, 593-604 (2014).

51. Nahak, P. et al. Influence of lipid core material on physicochemical characteristics of an ursolic acid-loaded nanostructured lipid carrier: An attempt to enhance anticancer activity. Langmuir 32, 9816-9825 (2016).

52. Kumar, V. V. et al. Development and evaluation of nitrendipine loaded solid lipid nanoparticles: Influence of wax and glyceride lipids on plasma pharmacokinetics. Int. J. Pharm. 335, 167-175 (2007).

53. Giron, D., Draghi, M., Goldbronn, C., Pfeffer, S. \& Piechon, P. Study of the polymorphic behaviour of some local anesthetic drugs. J. Therm. Anal. 49, 913-927 (1997).

54. Khalkhali, M., Mohammadinejad, S., Khoeini, F. \& Rostamizadeh, K. Vesicle-like structure of lipid-based nanoparticles as drug delivery system revealed by molecular dynamics simulations. Int. J. Pharm. 559, 173-181 (2019).

55. Böttner, M. \& Winter, R. Influence of the local anesthetic tetracaine on the phase behavior and the thermodynamic properties of phospholipid bilayers. Biophys. J. 65, 2041-2046 (1993).

56. Pereira, A. K. V., Barbosa, R. M., Fernandes, M. A. C., Finkler, L. \& Finkler, C. L. L. Comparative analyses of response surface methodology and artificial neural networks on incorporating tetracaine into liposomes. Braz. J. Pharm. Sci. 56, 17808 (2020).

57. Hu, S., Zhao, T., Li, H., Cheng, D. \& Sun, Z. Effect of tetracaine on dynamic reorganization of lipid membranes. Biochim. Biophys. Acta Biomembr. 1862, 183351 (2020). 


\section{Acknowledgements}

We would like to thank the Brazilian funding agencies FAPESP (\#14/25372-0, \#14/14457-5), FAPEAM (S.R.C. fellowship) and INCT-Bioanalítica. The authors also thank Gatteffosé Foundation and Dhaymers Química Fina, for kindly supplying the lipids.

\section{Author contributions}

E.P., L.N.M.R., D.R.A.: conceptualization, writing—review and editing; S.R.C., G.H.R.S., V.A.G.: HPLC, DLS, NTA, DSC and release kinetics experiments; S.R.C., M.L.: cell viability tests. S.R.C., M.C.B., H.M.: factorial design, Raman imaging; A.C.S.A., L.N.M.R.: FTIR; F.Y., M.K.K.D.F., D.C., B.K.: SANS analysis; G.H.R.S., M.C.B., F.Y., M.K.K.D.F.: review and editing.

\section{Competing interests}

The authors declare no competing interests.

\section{Additional information}

Supplementary Information The online version contains supplementary material available at https://doi.org/ 10.1038/s41598-021-99743-6.

Correspondence and requests for materials should be addressed to E.P.

Reprints and permissions information is available at www.nature.com/reprints.

Publisher's note Springer Nature remains neutral with regard to jurisdictional claims in published maps and institutional affiliations.

(c) (i) Open Access This article is licensed under a Creative Commons Attribution 4.0 International License, which permits use, sharing, adaptation, distribution and reproduction in any medium or format, as long as you give appropriate credit to the original author(s) and the source, provide a link to the Creative Commons licence, and indicate if changes were made. The images or other third party material in this article are included in the article's Creative Commons licence, unless indicated otherwise in a credit line to the material. If material is not included in the article's Creative Commons licence and your intended use is not permitted by statutory regulation or exceeds the permitted use, you will need to obtain permission directly from the copyright holder. To view a copy of this licence, visit http://creativecommons.org/licenses/by/4.0/.

(C) The Author(s) 2021 